\title{
BOREL MEASURES AND HAUSDORFF DISTANCE
}

\author{
GERALD BEER AND LUZVIMINDA VILLAR
}

\begin{abstract}
In this article we study the restriction of Borel measures defined on a metric space $X$ to the nonempty closed subsets $\mathrm{CL}(X)$ of $X$, topologized by Hausdorff distance. We show that a $\sigma$-finite Radon measure is a Borel function on $\mathrm{CL}(X)$, and characterize those $X$ for which each outer regular Radon measure on $X$ is semicontinuous when restricted to $\operatorname{CL}(X)$. A number of density theorems for Radon measures are also presented.
\end{abstract}

1. Introduction. Let $\mathrm{CL}(X)$ (resp. $K(X)$ ) denote the collection of closed (resp. compact) nonempty subsets of a metric space $\langle X, d\rangle$. We denote the open $\varepsilon$-ball with center $a$ in $X$ by $S_{\varepsilon}[a]$, and the union of all such balls whose centers run over a subset $A$ of $X$ by $S_{\varepsilon}[A]$. Certainly the most familiar topology on $\mathrm{CL}(X)$ is the Hausdorff metric topology (cf. Castaing and Valadier [4] or Klein and Thompson $[\mathbf{9}])$, induced by the infinite valued metric

$$
h_{d}(E, F)=\inf \left\{\varepsilon: S_{\varepsilon}[E] \supset F \text { and } S_{\varepsilon}[F] \supset E\right\} .
$$

It is the purpose of this article to study the behavior of Borel measures restricted to $\mathrm{CL}(X)$ with respect to this topology.

With respect to properties of measures, we adopt the terminology of the fundamental survey article of Gardner and Pfeffer [7]. A countably additive measure $\mu$ defined on the Borel $\sigma$-algebra of a metric space $X$ will be called a Borel measure if it is locally finite: at each $x \in X$, there is a neighborhood $V$ of $x$ with $\mu(V)<\infty$. A Borel measure $\mu$ is called a Radon measure if it is inner regular by compact sets: for each Borel set $B, \mu(B)=\sup \{\mu(K): K \in K(X)$ and $K \subset B\}$; it is called outer regular if for each Borel set $B, \mu(B)=\inf \{\mu(V): V$ open and $B \subset V\}$. Of course, a Borel measure is automatically outer regular at each compact set, and compact sets have finite measure. Although outer regular Radon measures have been called regular Borel measures in the widely read graduate texts of Halmos [8] and Rudin [16], this terminology remains nonstandard among researchers in measure theory.

In addition to [7], an earlier paper of Gardner [6] serves as a good second reference on Borel measures. Still, Chapters 8,9 , and 18 of [15] contain within them all that we need here. We shall use results from these chapters freely, and at this time single out two facts for special attention (cf. Exercises 9-2 and 9-3 of [15]).

LEMMA 1.1. Let $\left\langle x_{n}\right\rangle$ be a sequence in $X$ with distinct terms. Then the counting measure determined by $E=\left\{x_{n}: n \in Z^{+}\right\}$on the power set of $X$, defined by

Received by the editors April 10, 1986 and, in revised form, April 23, 1987.

1980 Mathematics Subject Classification (1985 Revision). Primary 28C15, 54B20.

Key words and phrases. Borel measure, Radon measure, Hausdorff distance, free union of measures, UC space, Baire space, weakly Baire space, Vietoris topology. 
$\mu(A)=$ the cardinality of $A \cap E$, is locally finite, and is hence an outer regular Radon measure, if and only if $\left\langle x_{n}\right\rangle$ has no cluster point.

LEMMA 1.2. Suppose $\sum_{n=1}^{\infty} \alpha_{n}$ is a convergent series of real numbers, and $\left\langle x_{n}\right\rangle$ is a sequence in $X$ with distinct terms. Then the assignment

$$
\mu(A)=\sum_{n=1}^{\infty} \alpha_{n} \chi_{A}\left(x_{n}\right)
$$

determines an outer regular Radon measure on $X$.

We set forth some additional notation and terminology. The set of limit points of $X$ will be denoted by $X^{\prime}$, and its set of isolated points by $I(X)$. If $A \subset X$, its closure and complement will be denoted by $\bar{A}$ and $A^{\mathrm{c}}$, respectively. The subset $A$ of $X$ is called discrete if its relative topology inherited from $X$ is discrete; $A$ is called $\varepsilon$-discrete if whenever $\left\{a_{1}, a_{2}\right\} \subset A$, we have $d\left(a_{1}, a_{2}\right) \geq \varepsilon$. By Zorn's lemma, a nonempty subset $A$ of $X$ will have for each positive $\varepsilon$ a maximal $\varepsilon$-discrete subset $E$. Such a set $E$ must satisfy $A \subset S_{\varepsilon}[E]$, whence $h_{d}(E, A) \leq \varepsilon$. We use this fact to prove the following lemma that will be used twice in the sequel.

LEMMA 1.3. Let $\mu$ be a Radon measure on a metric space $\langle X, d\rangle$. Suppose $F \subset X^{\prime}$ is nonempty, closed, and $\sigma$-finite. Then for each positive $\varepsilon$ and $\delta$ there exists a closed subset $A$ of $X$ with $h_{d}(A, F)<\varepsilon$ and $\mu(A)<\delta$.

Proof. By Lemma 9.4 of $[\mathbf{1 5}]$ there is a $\sigma$-compact subset $C$ of $F$ for which $\mu(F-C)=0$. Let $E$ be a maximal $\varepsilon / 3$-discrete subset of $F$, and set $B_{1}=E \cap C$ and $B_{2}=E \cap(F-C)$. Since the set $C$ is separable, $B_{1}$ must be countable. Write $B_{1}=\left\{b_{n}: n \in N\right\}$ where either $N$ is an initial segment of $Z^{+}$or $N=Z^{+}$. Now each neighborhood of $b_{n}$ contains infinitely many points, and by local finiteness of the measure, there exists a neighborhood within $S_{\varepsilon / 9}\left[b_{n}\right]$ of finite measure. By the countable additivity of $\mu$, we can find $x_{n}$ such that $d\left(b_{n}, x_{n}\right)<\varepsilon / 9$ and $\mu\left(\left\{x_{n}\right\}\right)<$ $2^{-n} \delta$. Note that $\left\{x_{n}: n \in N\right\}$ is $\varepsilon / 9$-discrete, so that $A=\left\{x_{n}: n \in N\right\} \cup B_{2}$ is closed. Also, $h_{d}(E, F) \leq \varepsilon / 3$ and $h_{d}(E, A) \leq \varepsilon / 9$ force $h_{d}(A, F)<\varepsilon$. Finally,

$$
\mu(A) \leq \mu\left(\left\{x_{n}: n \in N\right\}\right)+\mu(F-C)<\sum_{n=1}^{\infty} 2^{-n} \delta+0=\delta .
$$

2. Borel measurability of a Radon measure. Recall that an extended real valued function $f$ is called upper semicontinuous (u.s.c.) at a point $a$ of $X$ if whenever $\alpha>f(a)$, there exists a neighborhood $V$ of $a$ for which $f(V) \subset(-\infty, \alpha)$; equivalently, whenever $\left\langle x_{n}\right\rangle$ converges to $a$, we have $\limsup _{n \rightarrow \infty} f\left(x_{n}\right) \leq f(a)$. Dually, $f$ is called lower semicontinuous (l.s.c.) at $a$ if $-f$ is u.s.c. at $a$. Even for Lebesgue measure $\mu_{L}$ on the line, semicontinuity in either sense with respect to the Hausdorff metric on $\operatorname{CL}(X)$ fails. Lebesgue measure is not u.s.c. at the positive integers $Z^{+}$, for if $F_{n}=\bigcup_{j=1}^{\infty}[j-1 / n, j+1 / n]$, we have $\lim _{n \rightarrow \infty} h_{d}\left(F_{n}, Z^{+}\right)=$ $\lim _{n \rightarrow \infty} 1 / n=0$, but $\mu_{L}\left(Z^{+}\right)=0<\infty=\limsup _{n \rightarrow \infty} \mu_{L}\left(F_{n}\right)$. On the other hand, lower semicontinuity fails at each compact set of positive measure, for each one is the $h_{d}$-limit of a sequence of finite sets. As a special case of a more general result, we shall see that Lebesgue measure when restricted to $\mathrm{CL}(R)$ is however the limit of a sequence of u.s.c. measures. 
LEMMA 2.1. Let $\mu$ be a Borel measure on a metric space $\langle X, d\rangle$, and let $K \in$ $K(X)$. For each $F \in \mathrm{CL}(X)$, set $\lambda(F)=\mu(F \cap K)$. Then $\lambda$ is $h_{d}$-u.s.c. on $\mathrm{CL}(X)$.

Proof. Fix $F \in \mathrm{CL}(X)$. We consider two cases: (i) $F \cap K=\varnothing$; (ii) $F \cap K \neq \varnothing$. In the first case, there exists $\delta>0$ for which $S_{\delta}[F] \cap K=\varnothing$. Thus, if $h_{d}(F, E)<\delta$, we have $\lambda(E)=\mu(E \cap K) \leq \mu\left(S_{\delta}[F] \cap K\right)=0$, whence $\lambda$ is actually continuous at $F$. In the second case let $\left\langle F_{n}\right\rangle$ be a sequence in $\mathrm{CL}(X)$ convergent to $F$. Clearly, if $\left\langle n_{k}\right\rangle$ is an increasing sequence of positive integers and $x_{k} \in F_{n_{k}}$ for each $k$, then each cluster point of $\left\langle x_{k}\right\rangle$ must lie in $F$. By the compactness of $K$, for each $\delta>0$, it follows that $S_{\delta}[F \cap K]$ contains $F_{n} \cap K$ eventually. If $\lambda(F)=\infty$, then $\lambda$ is automatically $h_{d}$-u.s.c. at $F$. Otherwise, let $\alpha>\mu(F \cap K)$ be arbitrary. By local finiteness, there exists an open neighborhood $V$ of $F \cap K$ with $\mu(F \cap K) \leq \mu(V)<\alpha$. By the compactness of $F \cap K$, the open set contains $S_{\delta}[F \cap K]$ for some $\delta$. Since $S_{\delta}[F \cap K]$ contains $F_{n} \cap K$ eventually, we have

$$
\limsup _{n \rightarrow \infty} \lambda\left(F_{n}\right)=\limsup _{n \rightarrow \infty} \mu\left(F_{n} \cap K\right) \leq \mu(V)<\alpha .
$$

We conclude that $\limsup _{n \rightarrow \infty} \lambda\left(F_{n}\right) \leq \lambda(F)$.

The main result of this section bears on a question of Wilansky [18].

THEOREM 2.2. Let $\mu$ be a $\sigma$-finite Radon measure on a metric space $\langle X, d\rangle$. Then the restriction of $\mu$ to $\left\langle\mathrm{CL}(X), h_{d}\right\rangle$ is a Borel function.

PROOF. By Lemma 9.4 of [15], there exists an increasing sequence $\left\langle K_{n}\right\rangle$ in $K(X)$ with $\mu\left(X-\bigcup_{n=1}^{\infty} K_{n}\right)=0$. For each $n \in Z^{+}$and each $F \in \operatorname{CL}(X)$, let $\lambda_{n}(F)=\mu\left(F \cap K_{n}\right)$. By Proposition 8.5 of [15], we have $\mu(F)=\lim _{n \rightarrow \infty} \lambda_{n}(F)$; so, by Lemma $2.1, \mu$ restricted to $\mathrm{CL}(X)$ is a limit of an increasing sequence of $h_{d}$-u.s.c. functions and is thus of (extended) Baire class two.

3. Upper semicontinuity. We have seen that an outer regular Radon measure need not be $h_{d}$-u.s.c. on $\mathrm{CL}(X)$. However, there are certain closed sets at which such a measure must be $h_{d}$-u.s.c. We indicate these here, and characterize those $X$ for which each regular measure is $h_{d}$-u.s.c. on $\mathrm{CL}(X)$. The proof of the next fact follows immediately from the monotonicity of a measure and the definition of Hausdorff distance.

LEMMA 3.1. Let $\mu$ be a measure on the Borel $\sigma$-algebra of $\langle X, d\rangle$, and let $F \in \mathrm{CL}(X)$. Then $\mu$ is $h_{d}$-u.s.c. at $F$ if and only if $\lim _{n \rightarrow \infty} \mu\left(S_{1 / n}[F]\right)=\mu(F)$.

THEOREM 3.2. Let $\mu$ be a measure on the Borel $\sigma$-algebra of $\langle X, d\rangle$.

(i) If $\mu$ is locally finite, then $\mu$ is $h_{d}$-u.s.c. at each $K \in K(X)$.

(ii) If $F \in \mathrm{CL}(X)$ and $\mu\left(F^{\mathrm{c}}\right)<\infty$, then $\mu$ is $h_{d}$-u.s.c. at $F$.

PROOF. If $K \in K(X)$, then each open neighborhood of $K$ contains $S_{1 / n}[K]$ for some $n$, whence $\mu$ is u.s.c. at $K$ by Lemma 3.1 and local finiteness of $\mu$. On the other hand, if $\mu\left(F^{\mathrm{c}}\right)<\infty$, then for each $n, \mu\left(S_{1 / n}[F]-F\right)<\infty$. By Proposition 8.6 of [15],

$$
0=\mu(\varnothing)=\mu\left(\bigcap_{n=1}^{\infty}\left(S_{1 / n}[F]-F\right)\right)=\lim _{n \rightarrow \infty} \mu\left(S_{1 / n}[F]-F\right) .
$$

As a result, $\lim _{n \rightarrow \infty} \mu\left(S_{1 / n}[F]\right)=\mu(F)$ by additivity of $\mu$. 
An important class of outer regular Radon measures are finite Borel measures on Polish spaces (cf. [14]). By Theorem 3.2, each such is $h_{d}$-u.s.c. on CL $(X)$. We next characterize those closed subsets of an arbitrary metric space $X$ at which each outer regular Borel measure on $X$ is $h_{d}$-u.s.c.

THEOREM 3.3. Let $\langle X, d\rangle$ be a metric space and let $F \in \mathrm{CL}(X)$. The following are equivalent:

(i) Each outer regular Borel measure on $X$ is $h_{d}$-u.s.c. at $F$.

(ii) Whenever $E$ is a closed subset of $X$ disjoint from $F$, then $\inf \{d(x, y): x \in$ $E, y \in F\}>0$.

PROOF. (i) $\rightarrow$ (ii). If (ii) fails, then there exists a sequence $\left\langle x_{n}\right\rangle$ with distinct terms in $E$ such that for each $n, d\left(x_{n}, F\right)<1 / n$. Clearly, $\left\langle x_{n}\right\rangle$ can have no cluster point, for $E \cap F=\varnothing$. By Lemma 1.1, the counting measure $\mu$ for $\left\{x_{n}: n \in Z^{+}\right\}$ is outer regular. However, by Lemma $3.1, \mu$ fails to be $h_{d}$-u.s.c. at $F$ because for each $n$, the set $S_{1 / n}[F]$ contains infinitely many $x_{n}$.

(ii) $\rightarrow$ (i). Let $V$ be an open neighborhood of $F$. By (ii), there exists $\delta>0$ such that $\inf \left\{d(x, y): x \in V^{\mathrm{c}}, y \in F\right\}>\delta$. We conclude that $S_{\delta}[F] \subset V$, so that Lemma 3.1 guarantees the upper semicontinuity of each outer regular Borel measure at $F$.

As an immediate corollary of Theorem 3.3, we have

THEOREM 3.4. Let $\langle X, d\rangle$ be a metric space. The following are equivalent:

(i) Each outer regular Borel measure on $X$ is $h_{d}$-u.s.c. on $\mathrm{CL}(X)$.

(ii) Whenever $E$ and $F$ are disjoint members of $\mathrm{CL}(X)$, we have

$$
\inf \{d(x, y): x \in E, y \in F\}>0 \text {. }
$$

The class of spaces satisfying condition (ii) of Theorem 3.4 is well studied and properly contains the compact spaces. In the literature, such spaces have been called UC spaces, Lebesgue spaces, and Atsuji spaces (cf. [2, 13, and 17]). The first name reflects the fact that these are precisely the spaces on which each continuous function is uniformly continuous; the second has been used because these are also the spaces for which each open cover has a Lebesgue number. The last name acknowledges Atsuji's fundamental paper [1], containing the first extensive list of their characterizations. An analog of Theorem 3.4 characterizes the complete spaces, as follows.

THEOREM 3.5. Let $\langle X, d\rangle$ be a metric space. The following are equivalent:

(i) Each outer regular Borel measure on $X$ is $h_{d}$-u.s.c. on $T B(X)$, the collection of closed totally bounded subsets of $X$.

(ii) $\langle X, d\rangle$ is complete.

ProOf. (i) $\rightarrow$ (ii). Suppose $X$ is not complete; then there exists a Cauchy sequence $\left\langle x_{n}\right\rangle$ in $X$ with distinct terms with no cluster point. Set $F=\left\{x_{2 k}: k \in Z^{+}\right\}$ and let $\mu$ be the counting measure for $\left\{x_{2 k-1}: k \in Z^{+}\right\}$. (See Lemma 1.1.) For each $n \in Z^{+}$let $F_{n}=\left\{x_{2 k}: k \leq n\right\} \cup\left\{x_{2 k-1}: k>n\right\}$. Then $\left\langle F_{n}\right\rangle$ is a sequence in $T B(X)$ $h_{d}$-convergent to the totally bounded set $F$. Since $\mu(F)=0$ and $\mu\left(F_{n}\right)=\infty$ for all $n$, the measure is not $h_{d}$-u.s.c. on $T B(X)$.

(ii) $\rightarrow$ (i). Each closed and totally bounded subset of a complete space is compact; so, (i) follows from (ii) and Theorem 3.2.

We remark in closing that "Borel" can be replaced by "Radon" in the statements of each of the last three theorems. 
4. Lower semicontinuity. In this section we produce sufficient conditions for a Radon measure to be lower semicontinuous at a fixed closed set $F$, which are also necessary provided $F$ is $\sigma$-finite.

LEMMA 4.1. Let $F$ be a nonempty closed subset of a metric space $\langle X, d\rangle$, and let $\mu$ be a Radon measure on $X$. If $\mu(F)=\mu(F \cap I(X))$, then $\mu$ is $h_{d}$-l.s.c. at $F$.

ProOF. Let $\alpha<\mu(F)$ be arbitrary. By the inner regularity of $\mu$ at the $G_{\delta}$-set $F \cap I(X)$, and the fact that $[F \cap I(X)]^{\prime} \subset F-I(X)$, we have

$$
\mu(F)=\sup \{\mu(K): K \subset F \cap I(X), K \text { finite }\} .
$$

Choose a finite subset $\left\{x_{1}, \ldots, x_{n}\right\}$ of $F \cap I(X)$ with $\mu\left(\left\{x_{1}, \ldots, x_{n}\right\}\right)>\alpha$. Since each $x_{j}$ is isolated, there exists $\delta>0$ for which $S_{\delta}\left[x_{j}\right]=\left\{x_{j}\right\}$ for $j=1, \ldots, n$. Now if $h_{d}(E, F)<\delta$, the set $E$ must contain $\left\{x_{1}, \ldots, x_{n}\right\}$, whence $\mu(E)>\alpha$.

We were unable to find a simple counterexample showing that $\mu(F)=$ $\mu(F \cap I(X))$ is not necessary for $h_{d}$-lower semicontinuity of an outer regular Radon measure $\mu$ at $F$. Our construction involves the free union of an uncountable family of outer regular Radon measures. Suppose $\left\{X_{i}: i \in I\right\}$ is a disjoint family of Hausdorff spaces, and $\mu_{i}$ is outer regular and Radon on $X_{i}$ for each $i \in I$. Then the following set function always defines a Radon measure on the free union (topological sum) [5] $X=\sum_{i \in I} X_{i}$ of the spaces:

$$
\mu(A)=\sup \left\{\sum_{i \in J} \mu_{i}\left(A \cap X_{i}\right): J \text { finite, } J \subset I\right\} .
$$

However, $\mu$ need not be outer regular; in particular, $\mu$ is not outer regular if our collection of measure spaces consists of uncountably many copies of the line with Lebesgue measure. It can be shown [3] that outer regularity of $\mu$ is characterized by this condition: the set of indices $i$ for which the support of $\mu_{i}$ contains at least one point of measure zero is countable. Our counterexample involves a measure space of this type. Let $Q$ denote the rationals, and let $X=Q \times[0,1]$, equipped with the metric $d$ defined by

$$
d\left(\left(\alpha_{1}, \beta_{1}\right),\left(\alpha_{2}, \beta_{2}\right)\right)= \begin{cases}\min \left\{1,\left|\alpha_{1}-\alpha_{2}\right|\right\} & \text { if } \beta_{1}=\beta_{2} \\ 1 & \text { otherwise }\end{cases}
$$

Clearly, $X$ so metrized is the free union of uncountably many copies of the rationals with the usual topology. Let $\left\langle q_{n}\right\rangle$ be a one-to-one enumeration of $Q$, and let $\beta \in$ $[0,1]$ be fixed. By Lemma 1.2, the set function $\mu_{\beta}$ on $Q \times\{\beta\}$ defined by $\mu_{\beta}(A)=$ $\sum\left\{2^{-n}:\left(q_{n}, \beta\right) \in A\right\}$ is outer regular and Radon, whence by our remarks, the free union $\mu$ of $\left\{\mu_{\beta}: 0 \leq \beta \leq 1\right\}$ is also such a measure on $X$ because each point of $X$ has positive measure. Now let $F=\{0\} \times[0,1]$. Since $F$ is uncountable, $\mu(F)=\infty$, again because each point of $X$ has positive measure. Since $I(X)=\varnothing$, it is evident that $\mu(F) \neq \mu(F \cap I(X))$. Still, $\mu$ is l.s.c. at $F$, for if $h_{d}(E, F)<1$, then the set $E$ must meet $Q \times\{\beta\}$ for each $\beta \in[0,1]$. As a result, $E$ is uncountable and $\mu(E)=\infty$.

THEOREM 4.2. Let $\mu$ be a Radon measure on a metric space $\langle X, d\rangle$, and let $F \in \mathrm{CL}(X)$ be such that $F \cap X^{\prime}$ is $\sigma$-finite. The following are equivalent:

(i) $\mu$ is $h_{d}$-l.s.c. at $F$.

(ii) $\mu(F)=\mu(F \cap I(X))$. 
ProOF. The implication (ii) $\rightarrow$ (i) is established by Lemma 4.1. Conversely, suppose (ii) fails. Then both $\mu(F \cap I(X))<\infty$ and $\mu\left(F \cap X^{\prime}\right)>0$ hold. Pick $\delta$ satisfying $0<\delta<\mu\left(F \cap X^{\prime}\right)$, and let $\alpha=\mu(F \cap I(X))+\delta$. We show that for each $\varepsilon>0$, there exists $E \in \mathrm{CL}(X)$ with $h_{d}(E, F)<\varepsilon$ and $\mu(E)<\alpha$, which implies that (i) fails. Since $F \cap X^{\prime}$ is $\sigma$-finite, by Lemma 1.3, there exists $A \in \operatorname{CL}(X)$ with $h_{d}\left(A, F \cap X^{\prime}\right)<\varepsilon$ and $\mu(A)<\delta$. Let $B$ be a maximal $\varepsilon / 2$-discrete subset of $F \cap I(X)$. The set $E=A \cup B$ is closed and

$$
h_{d}(A \cup B, F) \leq \max \left\{h_{d}\left(A, F \cap X^{\prime}\right), h_{d}(B, \overline{F \cap I(X)})\right\}<\varepsilon .
$$

Finally

$$
\mu(E) \leq \mu(A)+\mu(B)<\delta+\mu(F \cap I(X))=\alpha .
$$

THEOREM 4.3. Let $\langle X, d\rangle$ be a metric space and let $F \in \mathrm{CL}(X)$. The following are equivalent:

(i) Each Radon measure $\mu$ on $X$ is $h_{d}$-l.s.c. at $F$.

(ii) $F \subset I(X)$.

PROOF. Suppose (ii) fails. Let $\left\langle x_{n}\right\rangle$ be a sequence of distinct terms in $X$ convergent to some $a \in F$. The Radon measure given by $\mu(A)=\chi_{A}(a)$ is not $h_{d}$-l.s.c. at $F$, for if $F_{n}=\left(F-S_{1 / n}[a]\right) \cup\left\{x: d(x, a)=d\left(x_{n}, a\right)\right\}$, then $\lim _{n \rightarrow \infty} h_{d}\left(F_{n}, F\right)=0$. Thus (i) fails. Lemma 4.1 yields (ii) $\rightarrow$ (i).

THEOREM 4.4. Let $\langle X, d\rangle$ be a metric space. The following are equivalent:

(i) $X=I(X)$.

(ii) Each Radon measure on $X$ is $h_{d}$-l.s.c. on $\mathrm{CL}(X)$.

PROOF. (i) $\rightarrow$ (ii). This is immediate from Lemma 4.1 or Theorem 4.3.

(ii) $\rightarrow$ (i). Suppose $X$ has a limit point $a$. Then the measure defined by $\mu(A)=$ $\chi_{A}(a)$ is a Radon measure that fails to be $h_{d}$-l.s.c. at $\{a\}$.

THEOREM 4.5. Let $\langle X, d\rangle$ be a metric space. The following are equivalent:

(i) For some $\varepsilon>0, X$ is $\varepsilon$-discrete.

(ii) Each Radon measure on $X$ is $h_{d}$-continuous on $\mathrm{CL}(X)$.

ProOF. (i) $\rightarrow$ (ii). Condition (i) implies that $h_{d}$ yields the discrete topology on $\mathrm{CL}(X)$.

(ii) $\rightarrow$ (i). Suppose (ii) holds; then by Theorem 4.4, $X=I(X)$. If (i) fails, for each $n \in Z^{+}$, we can find isolated points $x_{n}$ and $y_{n}$ with $d\left(x_{n}, y_{n}\right)<1 / n$. Since all points are isolated, we may assume without loss of generality that the terms of $x_{1}, y_{1}, x_{2}, y_{2}, \ldots$ are all distinct. As a result, $E=\left\{x_{n}: n \in Z^{+}\right\}$and $F=\left\{y_{n}: n \in Z^{+}\right\}$are disjoint closed sets that do not lie a positive distance apart so that $X$ is not a UC space. We conclude that $X$ admits a Radon measure that is not $h_{d}$-u.s.c., contradicting (ii).

5. Density theorems. Here, we address these questions: given a Radon measure on a metric space $\langle X, d\rangle$,

(1) When are the closed sets of measure zero $h_{d}$-dense in $\mathrm{CL}(X)$ ?

(2) When are the closed sets of finite measure $h_{d}$-dense in CL $(X)$ ?

The answer to (1) involves a class of spaces somewhat larger than the Baire spaces (recall [5] that $X$ is called Baire if the intersection of each sequence of dense open subsets of $X$ is again dense). 
DEFinition. A topological space $\langle X, d\rangle$ is called weakly Baire if each open dense-in-itself subset is uncountable.

It is easy to check that $X$ is Baire if and only if each open dense-in-itself subset is of second category in $X$. Now if $V$ is an open dense-in-itself subset of a Hausdorff space, each singleton subset of $V$ must be a nowhere dense subset of $X$. As a result, if $X$ is Hausdorff and Baire, and $V$ is an open dense-in-itself subset, then $V$ must be uncountable; so, $X$ is weakly Baire. However, a weakly Baire space need not be Baire. For example, $Q \times R$ as a subspace of the plane is a weakly Baire space that is a first category space. The subspace of $l_{\infty}$ consisting of those sequences that are zero eventually is another such space. Krom [10] has presented a metric Baire space $X$ for which $X^{2}$ is not Baire. But weakly Baire spaces are closed under arbitrary products. For the space $X$ of Krom, McCoy [11] has shown that $\left\langle K(X), h_{d}\right\rangle$ fails to be Baire. We leave it to the reader to show that the hyperspace is still weakly Baire, resulting in another example of a weakly Baire space that is not Baire.

LEMMA 5.1. Let $\mu$ be a Radon measure on a metric space $\langle X, d\rangle$. Suppose that each nonempty open subset of $X$ contains a point of measure zero. Then for each $F \in \mathrm{CL}(X)$ and $\varepsilon>0$, there exists $\delta>0$ and a $\delta$-discrete set $E$ with $h_{d}(F, E)<\varepsilon$ and $\mu(E)=0$.

ProOF. Let $A$ be a maximal $\varepsilon / 3$-discrete subset of $F$. For each $a \in A$ we may choose $x_{a}$ in the open set $S_{\varepsilon / 9}[a]$ with $\mu\left(\left\{x_{a}\right\}\right)=0$. Now $E=\left\{x_{a}: a \in A\right\}$ is $\varepsilon / 9$-discrete, and $S_{\varepsilon / 2}[E] \supset F$ and $S_{\varepsilon / 9}[F] \supset E$ hold. Thus, $h_{d}(E, F)<\varepsilon$. Finally, by inner regularity, $E$ has measure zero because each compact subset of $E$ is finite.

THEOREM 5.2. Let $\mu$ be a Radon measure on a metric space $\langle X, d\rangle$. The following are equivalent:

(i) $\{x: \mu(\{x\})=0\}$ is dense in $X$.

(ii) The closed sets of measure zero are dense in $\left\langle\mathrm{CL}(X), h_{d}\right\rangle$.

(iii) The finite sets of measure zero are dense in $\left\langle K(X), h_{d}\right\rangle$.

(iv) The compact sets of measure zero are dense in $\left\langle K(X), h_{d}\right\rangle$.

ProOF. The implication (i) $\rightarrow$ (ii) is immediate from Lemma 5.1. Suppose (ii) holds, and $K \in K(X)$ and $\varepsilon>0$ are arbitrary. Since $K \in \mathrm{CL}(X)$, there exists by (ii) $E \in \mathrm{CL}(X)$ with $\mu(E)=0$ and $h_{d}(K, E)<\varepsilon / 2$. Let $A$ be a finite subset of $K$ with $K \subset S_{\varepsilon / 2}[A]$. For each $a \in A$, choose $x_{a} \in E$ with $d\left(a, x_{a}\right)<\varepsilon / 2$. Then $F=\left\{x_{a}: a \in A\right\}$ is a finite set of measure zero satisfying $h_{d}(K, F)<\varepsilon$. The proof of (iii) $\rightarrow$ (iv) is trivial. Finally, suppose (iv) holds. Since $\{a\} \in K(X)$ for each $a \in X$, the density of the sets of measure zero in $K(X)$ forces $S_{\varepsilon}[a]$ to contain a compact set, and therefore a point, of measure zero for each positive $\varepsilon$. Thus, (i) holds.

COROLlaRY 5.3. Let $\langle X, d\rangle$ be a metric space and let $\mu$ be a Borel measure on $X$ for which $\{x: \mu(\{x\})=0\}$ is dense in $X$. Then $\{K: K \in K(X)$ and $\mu(K)=0\}$ forms a dense $G_{\delta}$-set in $\left\langle K(X), h_{d}\right\rangle$.

ProOF. By the upper semicontinuity of $\mu$ on $K(X)$, for each $n \in Z^{+}$the set $\{K: K \in K(X)$ and $\mu(K)<1 / n\}$ is $h_{d}$-open in $K(X)$. The density of sets of measure zero holds in $K(X)$ without inner regularity, and the result follows.

Evidently, whenever $\mu$ is a diffused Radon measure, then the sets of measure zero are dense in $\mathrm{CL}(X)$ (we remark that each outer regular diffused Radon measure 
is necessarily $\sigma$-finite (see, e.g., p. 245 of $[\mathbf{1 5}]$ or, more generally, Corollary 13.4 of $[7]))$. A necessary condition for each nonempty open subset of $X$ to contain a point of measure zero is that each isolated point of $X$ have measure zero; by inner regularity, this is equivalent to requiring that $\mu(I(X))=0$, i.e., that $\operatorname{supp} \mu \subset X^{\prime}$. This condition is also sufficient for a Radon measure when $X$ is weakly Baire. More precisely, we have

THEOREM 5.4. Let $\langle X, d\rangle$ be a metric space. The following are equivalent:

(i) $X$ is weakly Baire.

(ii) Whenever $\mu$ is a Radon measure on $X$ with $\mu(I(X))=0$, then the sets of measure zero are $h_{d}$-dense in $\mathrm{CL}(X)$.

PROOF. (i) $\rightarrow$ (ii). Let $\mu$ be Radon with $\mu(I(X))=0$; we show that each nonempty open subset $V$ of $X$ contains a point of measure zero. If $V \cap \overline{I(X)} \neq \varnothing$, then $V \cap I(X) \neq \varnothing$, and we are done. Otherwise $V=V-\overline{I(X)}$, so that $V$ is dense-in-itself. Fix $a \in V$; since $X$ is weakly Baire and each open subset of a densein-itself set is dense-in-itself, the ball $S_{\varepsilon}[a]$ contains uncountably many points for each positive $\varepsilon$. By local finiteness, $\mu\left(S_{\varepsilon}[a]\right)$ is finite for some $\varepsilon$ with $S_{\varepsilon}[a] \subset V$; so, by countable additivity of $\mu$, this ball contains a point of measure zero.

(ii) $\rightarrow$ (i). Suppose that (i) fails, i.e., $X$ contains a countable open dense-in-itself subset $V$. Sequence $V$ in a one-to-one fashion by $\left\langle a_{n}\right\rangle$. By Lemma 1.2, the weighted counting measure $\mu(A)=\sum 2^{-n} \chi_{A}\left(a_{n}\right)$ is a Radon measure on $X$, and it assigns measure zero to each isolated point of $X$. Clearly, $V$ contains no point of measure zero. By Theorem 5.2, we conclude that the sets of measure zero are not $h_{d}$-dense in $\mathrm{CL}(X)$. Thus, (ii) fails.

THEOREM 5.5. Let $\langle X, d\rangle$ be a metric space. The following are equivalent:

(i) Each open subset of $X$ is uncountable.

(ii) $X$ is weakly Baire and $I(X)=\varnothing$.

(iii) Whenever $\mu$ is a Radon measure on $X$, then the closed subsets of measure zero are $h_{d}$-dense in $\mathrm{CL}(X)$.

PROOF. The equivalence of (i) and (ii) is obvious, and (ii) implies (iii) by Theorem 5.4. Now suppose (iii) holds. By Theorem 5.4, $X$ must be weakly Baire. Also, if $X$ had an isolated point $p$, then $\mu(A)=\chi_{A}(p)$ would be a Radon measure on $X$ for which $\{p\}$ would not be in the $h_{d}$-closure of the sets of measure zero, in violation of (iii). Thus, (ii) holds.

THEOREM 5.6. Let $\mu$ be a Radon measure on a metric space $\langle X, d\rangle$. Suppose that either $\mu$ is $\sigma$-finite, or $X$ is weakly Baire. The following are equivalent:

(i) For each $\varepsilon>0$ there exists a Borel set $B$ of finite measure with $I(X) \subset S_{\varepsilon}[B]$.

(ii) $\{F: F \in \mathrm{CL}(X)$ and $\mu(F)<\infty\}$ is $h_{d}$-dense in $\mathrm{CL}(X)$.

PROOF. (ii) $\rightarrow$ (i). If $I(X)=\varnothing$, let $B$ be any singleton subset of $X$. Otherwise, choose a closed set of finite measure $B$ for which $h_{d}(\overline{I(X)}, B)<\varepsilon$.

(i) $\rightarrow$ (ii). First assume $\mu$ is $\sigma$-finite. Fix $F \in \mathrm{CL}(X)$ and let $\varepsilon$ be positive. By Lemma 1.3, there exists a closed set $A$ of finite measure with $h_{d}\left(A, F \cap X^{\prime}\right)<\varepsilon$. Choose a Borel set $B$ of finite measure with $I(X) \subset S_{\varepsilon / 4}[B]$, and let $C$ be a maximal $\varepsilon / 4$-discrete subset of $B$. Then

$$
A^{*}=\left\{x: x \in C \text { and } S_{\varepsilon / 2}[x] \cap(F \cap I(X)) \neq \varnothing\right\}
$$


is an $\varepsilon / 4$-discrete set satisfying $S_{\varepsilon / 2}\left[A^{*}\right] \supset F \cap I(X)$ and $S_{\varepsilon / 2}[F \cap I(X)] \supset A^{*}$. As a result, the closed set $E=A \cup A^{*}$ has finite measure and satisfies $h_{d}(E, F)<\varepsilon$.

Now assume $X$ is weakly Baire. By condition (i), there exists for each $\varepsilon>0$ a Borel set $B$ of finite measure with $\overline{I(X)} \subset S_{\varepsilon}[B]$; so, arguing as in the $\sigma$-finite case with $I(X)$ replaced by $\overline{I(X)}$, for each $F \in \mathrm{CL}(X)$, the set $F \cap \overline{I(X)}$ can be approximated in Hausdorff distance by closed sets of finite measure. Also, the proof of Lemma 5.1 shows that $F-\overline{I(X)}$ can be approximated by closed sets of measure zero because each point of $F-\overline{I(X)}$ is a condensation point of $X$ and is, therefore, a limit point of $\{x: \mu(\{x\})=0\}$. Condition (ii) now follows in the obvious way.

Condition (i) does not force condition (ii) without some additional assumptions on $\mu$ and/or on the space $X$. To see this, we return to the free union $X$ of uncountably many copies of $Q$ as presented in $\S 4$. Since $I(X)=\varnothing$ for this metric space, (i) holds vacuously. However, no closed subset of $X$ which is intersected by uncountably many horizontal lines can be approximated by closed sets of finite measure in Hausdorff distance.

6. Borel measures and the Vietoris topology. Ranking second in familiarity among topologies on closed subsets is the Vietoris (or finite) topology $\tau_{V}$. Basic facts about this topology can be found in the fundamental paper of Michael [12] or in [9]. Most simply described, this topology has as a subbase all sets of the form

$$
\begin{aligned}
& V^{+}=\{F: F \in \mathrm{CL}(X) \text { and } F \subset V\}, \\
& V^{-}=\{F: F \in \mathrm{CL}(X) \text { and } F \cap V \neq \varnothing\},
\end{aligned}
$$

where $V$ is an arbitrary open subset of $X$. Although this topology agrees with the $h_{d^{-}}$-topology on $K(X)$, it is generally neither stronger nor weaker than the $h_{d^{-}}$ topology on $\mathrm{CL}(X)$. Specifically, $\tau_{V}$ includes the $h_{d}$-topology if and only if $\langle X, d\rangle$ is totally bounded, whereas the reverse inclusion holds if and only if $\langle X, d\rangle$ is a UC space. (cf. Lemma 3.2 of [12]). Of course, this topology makes sense when $X$ is an arbitrary topological space, and the behavior of Borel measures with respect to it is no more difficult to describe in this broader context. It turns out that the theory is substantially less rich than that developed in the previous sections. We outline some results here, under the assumption that $X$ is Hausdorff.

First, it is easy to see that each outer regular Borel measure is $\tau_{V}$-u.s.c. on all of $\mathrm{CL}(X)$, for if $V$ is open and $\mu(V)<\alpha$, then $F \in V^{+}$implies $\mu(F)<\alpha$. Also, the sets of finite measure for each Borel measure on $X$ are always dense in $\left\langle\mathrm{CL}(X), \tau_{V}\right\rangle$, for the finite subsets of $X$ are dense in $\left\langle\mathrm{CL}(X), \tau_{V}\right\rangle$ (cf. Proposition 2.1.6 of [9]). It is not hard to show that Theorem 4.2 goes through without the $\sigma$-finiteness assumption, whereas in the statement of Theorem $5.2, K(X)$ may be replaced by $\operatorname{CL}(X)$ wherever it appears.

\section{REFERENCES}

1. M. Atsuji, Uniform continuity of continuous functions of metric spaces, Pacific J. Math. 8 (1958), 11-16.

2. G. Beer, Metric spaces on which continuous functions are uniformly continuous and Hausdorff distance, Proc. Amer. Math. Soc. 95 (1985), 653-658.

3. G. Beer and L. Villar, When is a free union of regular Borel measures regular?, Amer. Math. Monthly 94 (1987), 443445. 
4. C. Castaing and M. Valadier, Convex analysis and measurable multifunctions, Lecture Notes in Math., Vol. 580, Springer-Verlag, Berlin, 1977.

5. J. Dugundji, Topology, Allyn and Bacon, Boston, Mass., 1966.

6. R. Gardner, The regularity of Borel measures, Measure Theory Oberwolfach 1981, Lecture Notes in Math., Vol. 945, Springer-Verlag, New York, 1982.

7. R. Gardner and W. Pfeffer, Borel measures, Handbook of Set-Theoretic Topology (K. Kunen and J. Vaughan, Eds.), Elsevier, Amsterdam, 1984.

8. P. Halmos, Measure theory, Van Nostrand Reinhold, New York, 1950.

9. E. Klein and A. Thompson, Theory of correspondences, Wiley, New York, 1984.

10. M. Krom, Cartesian products of metric Baire spaces, Proc. Amer. Math. Soc. 42 (1973), 588594.

11. R. McCoy, Baire spaces and hyperspaces, Pacific J. Math. 58 (1975), 133-142.

12. E. Michael, Topologies on spaces of subsets, Trans. Amer. Math. Soc. 71 (1951), 152-182.

13. S. Nadler and T. West, A note on Lebesgue spaces, Topology Proc. 6 (1982), 363-369.

14. K. Parthasarathy, Probability measures on metric spaces, Academic Press, New York, 1967.

15. W. Pfeffer, Integrals and measure, Dekker, New York, 1977.

16. W. Rudin, Real and complex analysis, McGraw-Hill, New York, 1966.

17. W. Waterhouse, On UC spaces, Amer. Math. Monthly 72 (1965), 634-635.

18. A. Wilansky, Elementary problem \# E 3147, Amer. Math. Monthly 93 (1986), 400.

Department of Mathematics, California State University, los Angeles, CALIFORNIA 90032

Department of Mathematics, De la Salle University, 2401 Taft Avenue, Manila, The PhiLipPines 